\title{
Total Acquisition in Graphs
}

\author{
Timothy D. LeSaulnier, Noah Prince† \\ Paul S. Wenger $;$ Douglas B. West $\stackrel{\S}{\ddagger}$ Pratik Worah
}

November, 2011; revised April, 2013

\begin{abstract}
Let $G$ be a weighted graph in which each vertex initially has weight 1 . A total acquisition move transfers all the weight from a vertex $u$ to a neighboring vertex $v$, under the condition that before the move the weight on $v$ is at least as large as the weight on $u$. The (total) acquisition number of $G$, written $a_{t}(G)$, is the minimum size of the set of vertices with positive weight after a sequence of total acquisition moves.

Among connected $n$-vertex graphs, $a_{t}(G)$ is maximized by trees. The maximum is $\Theta(\sqrt{n \lg n})$ for trees with diameter 4 or 5 . It is $\lfloor(n+1) / 3\rfloor$ for trees with diameter between 6 and $\frac{2}{3}(n+1)$, and it is $\lceil(2 n-1-D) / 4\rceil$ for trees with diameter $D$ when $\frac{2}{3}(n+1) \leq D \leq n-1$. We characterize trees with acquisition number 1 , which permits testing $a_{t}(G) \leq k$ in time $O\left(n^{k+2}\right)$ on trees.

If $G \neq C_{5}$, then $\min \left\{a_{t}(G), a_{t}(\bar{G})\right\}=1$. If $G$ has diameter 2 , then $a_{t}(G) \leq$ $32 \ln n \ln \ln n$; we conjecture a constant upper bound. Indeed, $a_{t}(G)=1$ when $G$ has diameter 2 and no 4-cycle, except for four graphs with acquisition number 2.

Deleting one edge of an $n$-vertex graph cannot increase $a_{t}$ by more than $6.84 \sqrt{n}$, but we construct an $n$-vertex tree with an edge whose deletion increases it by more than $\frac{1}{2} \sqrt{n}$. We also obtain multiplicative upper bounds under products.
\end{abstract}

\section{Introduction}

Consider an army dispersed among many cities. We wish to consolidate the troops. Troops move only to neighboring occupied cities, and the number of troops in a move cannot exceed the number already at the destination. Can the troops all move to one city?

In another scenario, each person in a society starts with one vote. Person $A$ may conclude that friend $B$ who has acquired at least as many votes as $A$ has a better chance to win. In

\footnotetext{
*Mathematics Dept., University of Illinois, Urbana, IL; tlesaul2@illinois.edu.

†Illinois Mathematics and Science Academy, Aurora, IL; nprince@imsa.edu.

${ }^{\ddagger}$ Dept. of Mathematical and Statistical Sciences, University of Colorado Denver, Denver, CO, and School of Mathematical Sciences, Rochester Institute of Technology, Rochester, NY; paul . wenger@ucdenver . edu.

$\S$ Mathematics Depts., Zhejiang Normal University, Jinhua, China, and University of Illinois, Urbana, IL; west@math . uiuc.edu, supported in part by National Security Agency Grant H98230-10-0363.

IDept. of Computer Science, University of Chicago, Chicago, IL; pworah@cs .uchicago.edu.
} 
such a situation, $A$ can transfer all his votes to $B$. Is it possible for the society to elect a leader by one person acquiring all the votes?

We model such situations using graphs with vertex weights. Initially, each vertex has weight 1; let 1 denote this initial assignment. An acquisition move transfers some weight from a vertex $u$ to a neighboring vertex $v$, provided that before the move the weight on $v$ is at least the weight on $u$. The total weight is preserved. We want to concentrate the weight on the fewest vertices. Acquisition moves can be made until the set of vertices with positive weight is an independent set; the final independent set is the residual set.

Lampert and Slater [5] introduced acquisition in graphs, using acquisition moves that transfer all of the weight from a vertex to a neighbor. The total acquisition number, written $a_{t}(G)$, is the minimum possible size of the residual set after such acquisition moves (starting from distribution 1). We refer to a succession of total acquisition moves as an acquisition protocol. An acquisition protocol $\mathcal{A}$ on a graph $G$ is optimal if it starts with the weight assignment 1 and leaves positive weight on only $a_{t}(G)$ vertices.

Allowing flexibility in the amount of weight moved leads to variations. When an acquisition move may transfer any integer portion of the weight on a vertex, the minimum size of the residual set is the unit acquisition number $a_{u}(G)$. When it may transfer any positive amount of the weight on a vertex, the minimum size of the residual set is the fractional acquisition number $a_{f}(G)$. For results on $a_{u}$ and $a_{f}$, see $[9,12]$.

Lampert and Slater [5] proved that $a_{t}(G) \leq\lfloor(n+1) / 3\rfloor$ when $G$ is a connected $n$-vertex graph (for $n>1$ ), and this is sharp. They also observed that a vertex $v$ cannot acquire weight more than $2^{d(v)}$ and that no weight from $u$ can reach $v$ if the distance between them is greater than $d(v)$. Here $d_{G}(v)$ denotes the degree of vertex $v$ in a graph $G$, shortened to $d(v)$ when $G$ is understood. Slater and Wang [11] proved that testing $a_{t}(G)=1$ is NP-complete, and they provided a linear-time algorithm to compute $a_{t}(G)$ when $G$ is a caterpillar.

Later, Slater and Wang [10] introduced "competitive acquisition": a "Consolidator" C and an Adversary A alternately perform acquisition moves until no more are possible. The Consolidator wants the residual set to be small; the Adversary wants it large. The competitive acquisition number is the resulting size when $\mathrm{C}$ moves first and both play optimally. Slater and Wang [10] computed this for the $n$-vertex path $P_{n}$, for all $n$. The problem is studied in [7] for trees and complete bipartite graphs, under the name game acquisition.

In this paper we study only total acquisition, so we abbreviate "total acquisition number" to "acquisition number" but maintain the notation $a_{t}$. Deleting edges cannot reduce $a_{t}$, so $a_{t}$ is maximized among $n$-vertex connected graphs by trees. In Section 2 , we present a family of trees showing that for $k \geq 3$ and $D \geq 6$, the largest acquisition number among $n$ vertex graphs can be achieved by a tree with maximum degree $k$ and diameter $D$ whenever $n$ is between $3(k+D / 2)-10$ and about $3(k-2)^{\lfloor D / 4\rfloor}$; the lower bound is equivalent to $D \leq \frac{2}{3}(n+1)$. In this range, the family of extremal trees is much richer than the single $n$-vertex example given in [5]. Actually, our construction generates all $n$-vertex graphs with 
acquisition number $(n+1) / 3$ when $n \equiv 2 \bmod 3$ (except for the 5 -cycle); this is proved in [6]. For larger diameter, with $\frac{2}{3}(n+1) \leq D \leq n-1$, we show that the maximum of $a_{t}$ is $\lceil(2 n-1-D) / 4\rceil$.

Trees with diameter less than 6 have smaller acquisition numbers. Trivially, $a_{t}(T)=1$ when $T$ is a tree with diameter at most 3 . For $n$-vertex trees with diameter 4 and diameter 5 , we show in Section 3 that the maximum is $\Theta(\sqrt{n \lg n})$, where $\lg$ denotes $\log _{2}$. In fact, the maximum among $n$-vertex trees with diameter 4 is between $\sqrt{n \lg n}$ and $\sqrt{.5 n \lg n}$. We also characterize trees $T$ for which $a_{t}(T)=1$, which allows us to construct a polynomial-time algorithm to test $a_{t}(T) \leq k$ for any fixed positive integer $k$.

In Section 4, we give sufficient conditions for a graph to have acquisition number 1 . We show that, if $G \neq C_{5}$, then $a_{t}(G)$ or $a_{t}(\bar{G})$ is 1 , where $\bar{G}$ denotes the complement of $G$. Furthermore, if $\delta(G) \geq(|V(G)|-1) / 2$, then $a_{t}(G)=1$ (again, if $G \neq C_{5}$ ), and no smaller minimum degree is sufficient.

In Section 5, we consider graphs with diameter 2. We conjecture that $a_{t}(G)$ is bounded by an absolute constant for such graphs, perhaps by 2 . We prove that $a_{t}(G) \leq 32 \ln n \ln \ln n$ when $G$ has diameter 2 (the diameter of a graph is the maximum of the distances between vertices). If in addition $G$ has no 4-cycle, then $a_{t}(G)=1$ except for $C_{5}$, the Petersen graph, one graph with seven vertices, and one graph with 13 vertices. The exceptions with seven and 13 vertices are the polarity graphs of the projective planes of orders 2 and 3, and all four exceptions have acquisition number 2 .

In Section 6, we consider edge deletion. Deleting one edge in an $n$-vertex graph cannot increase the acquisition number by more than $6.84 \sqrt{n}$, but there is an $n$-vertex tree having an edge whose deletion increases the acquisition number by more than $\frac{1}{2} \sqrt{n}$. For the strong product $\otimes$ and cartesian product $\square$, we show in Section 7 that $a_{t}(G \otimes H) \leq a_{t}(G \square H) \leq$ $a_{t}(G) a_{t}(H)$ and ask whether there are infinitely many examples with $a_{t}(G \otimes H)<\frac{1}{2} a_{t}(G) a_{t}(H)$.

In addition to the conjectures and open questions mentioned above, there are several directions for further research on total acquisition in graphs. Motivated by the sufficient conditions in Section 4 for acquisition number 1, it is natural to consider the more general problem of finding sufficient conditions for acquisition number at most $k$. For example, one can ask for the least such value of the minimum degree, the connectivity, or the minimum of $d(u)+d(v)$ over $u v \notin E(G)$ (known as an "Ore-type" condition). These questions also extend naturally to random graphs; what is the threshold edge-probability function for acquisition number at most $k$ ?

Alternatively, one can seek the maximum of $a_{t}(G)$ over other families of $n$-vertex graphs. For example, what is the maximum of $a_{t}(G)$ when $G$ is $k$-connected or when $\delta(G) \geq k$ ? Similarly, what is the behavior of the acquisition number of the random graph $G(n, p)$ with edge-probability $p(n)$ ? Of particular interest would be $p \sim \frac{\ln n}{n}$, the threshold for connectivity.

More generally, one can study the tradeoff between $a_{t}(G)$ and other parameters. In particular, for $n$-vertex graphs, how does $a_{t}(G)$ decrease as $\kappa(G)$ or $\delta(G)$ increases. Is $a_{t}(G) \kappa(G)$ 
or $a_{t}(G) \delta(G)$ bounded by a linear function of $|V(G)|$ ? Similarly, how does $a_{t}(G(n, p))$ decrease as $p \rightarrow 1$ ? The material of Section 2 can be viewed as exploring the tradeoff between diameter and acquisition number for trees.

We conclude this introduction with elementary observations about total acquisition. Let $\alpha(G)$ denote the maximum size of an independent set in $G$, and let $\gamma(G)$ denote the minimum size of a dominating set in $G$, where a dominating set is a vertex set $S$ such that every vertex outside $S$ has a neighbor in $S$.

Observation 1.1. For a graph $G$, the following statements hold:

(1) $a_{t}(G) \leq \alpha(G)$ and $a_{t}(G) \leq \gamma(G)$.

(2) The set of edges used in an acquisition protocol is acyclic, and each is used at most once.

(3) $a_{t}(G)=\min \left\{a_{t}(F): F \in \mathcal{F}\right\}$, where $\mathcal{F}$ is the set of spanning forests of $G$.

Proof. (1) An acquisition protocol can consolidate weight onto any maximal independent set or any minimal dominating set.

(2) Weight can never be moved to a vertex once it has weight 0 . Hence a cycle cannot be completed and an edge cannot be reused (and every acquisition protocol is finite).

(3) By (2), the set of edges used in an optimal acquisition protocol on $G$ is the edge set of a spanning forest. Deleting the unused edges does not change the residual set.

Example 1.2. With $P_{n}$ and $C_{n}$ denoting the $n$-vertex path and $n$-vertex cycle, $a_{t}\left(P_{n}\right)=$ $a_{t}\left(C_{n}\right)=\lceil n / 4\rceil$. As noted earlier, the weight on a vertex of degree $d$ cannot exceed $2^{d}$ when starting with weights 1 [5]. Hence the acquisition number of any graph with maximum degree 2 is at least $\lceil n / 4\rceil$. The vertices of a path or cycle with $n$ vertices can be covered by $\lceil n / 4\rceil$ paths with at most four vertices, each having acquisition number 1.

The acquisition problem generalizes to every graph $G$ with vertex weights; let $a_{t}(G)$ denote the minimum size of the residual set after an acquisition protocol starting with the weighted graph $G$. A cut-set in a graph $G$ is a set $S \subseteq V(G)$ such that $G-S$ is disconnected, where $G-S$ is the result of deleting the vertices of $S$ from $G$.

Observation 1.3. Let $S$ be a cut-set in a weighted graph $G$. If each vertex in $S$ has weight 0 , then $a_{t}(G)$ is at least the number of components of $G-S$ having positive total weight.

Proof. No weight can move to a vertex of weight 0, so no weight can move from one component of $G-S$ to another. 


\section{Extremal Trees}

Lampert and Slater [5] showed that $\lfloor(n+1) / 3\rfloor$ is the maximum of $a_{t}(G)$ over $n$-vertex graphs, when $n>1$. For $n \equiv 2 \bmod 3$, they provided a tree achieving this bound, but its maximum degree is $(n+1) / 3$ and its diameter is 6 . We construct a more general family of extremal trees. Note that since $a_{t}\left(P_{n}\right)=\lceil n / 4\rceil$, the bound is not sharp when $\Delta(G)=2$, where $\Delta(G)$ denotes the maximum vertex degree in $G$.

It is sometimes useful to view the initial weight on each vertex as a chip that moves from vertex to vertex under total acquisition moves. One can then follow a given chip to see where that weight goes during an acquisition protocol.

Lemma 2.1. Let $x$ and $y$ be vertices in a tree $T$. If the unique $x, y$-path in $T$ contains a vertex of degree 2 not adjacent to $x$ or $y$, then the initial weight from $x$ and $y$ cannot reach a common vertex via total acquisition moves.

Proof. Let $v$ be a vertex of degree 2 on the $x, y$-path. For the weight from $x$ and $y$ to reach the same vertex, vertex $v$ must be used. The first move involving $v$ transfers weight 1 to or from it, so this move cannot transfer the weight that was originally on $x$ or $y$. After this move, $v$ or one of its neighbors has weight 0 . By Observation 1.3, the weight from $x$ and $y$ cannot then reach the same vertex.

Theorem 2.2. Starting with $P_{5}$, let $\mathcal{T}$ be the family of trees constructed by iteratively growing a path with three edges from the neighbor of a leaf. If $T \in \mathcal{T}$, then $a_{t}(T)=(|V(T)|+1) / 3$.

Proof. We use induction on $j$, the number of augmentations. Each augmentation adds a path of length 3 through three new vertices, so $|V(T)|=3 j+5$. Each augmentation adds one new leaf, so $T$ has $j+2$ leaves. Initially, the central vertex of $P_{5}$ is a vertex of degree 2 separating the two leaves and adjacent to neither.

With each augmentation, the vertex $v$ at distance 2 from the new leaf $x$ is a vertex of degree 2 that separates $x$ from all earlier leaves. Since $v$ was made adjacent to a neighbor $w$ of an earlier leaf, it is not adjacent to any earlier leaf. Also, $w$ is not a vertex that was nonadjacent to all earlier leaves, so the vertices previously chosen to witness separation between leaves still have degree 2. By Lemma 2.1, $a_{t}(T) \geq j+2$.

Equality holds, because $a_{t}\left(P_{5}\right)=2$, and for $j>0$ the weight on each added path can be acquired to the central vertex among the three new vertices.

Corollary 2.3. For $k \geq 3$ and $D \geq 6$ with $D$ even, there is an n-vertex tree with maximum degree $k$, diameter $D$, and acquisition number $\lfloor(n+1) / 3\rfloor$ if $3(k+D / 2)-10 \leq n \leq n_{k, D}$, where

$$
n_{k, D}=\left\{\begin{array}{lll}
\frac{3}{2} D-1 & \text { for } k=3 \\
2+3(k-1) \frac{(k-2)^{(D-2) / 4}-1}{k-3} & \text { for } k>3 \text { and } D \equiv 2 & \bmod 4 \\
5+6(k-2) \frac{(k-2)^{(D-4) / 4}-1}{k-3} & \text { for } k>3 \text { and } D \equiv 0 & \bmod 4
\end{array}\right.
$$


Proof. Suppose first that $n \equiv 2 \bmod 3$. We construct such a tree $T$ in the family $\mathcal{T}$ of Theorem 2.2, which guarantees $a_{t}(T)=(n+1) / 3$.

To reach maximum degree $k$, begin with $P_{5}$ in which $v$ is the neighbor of a leaf, and augment at $v$ exactly $k-2$ times. Now the diameter is 6 , and $v$ has degree $k$. Next increase the diameter to $D$ by iteratively augmenting at the neighbor of a leaf on a longest path, $(D-6) / 2$ times. The resulting tree $T$ has diameter $D$, maximum degree $k$, and $3(k+D / 2)-10$ vertices, since there were $k-2+D / 2-3$ augmentations to $P_{5}$. Figure 1 shows such a construction for $k=6$ and $D=10$.

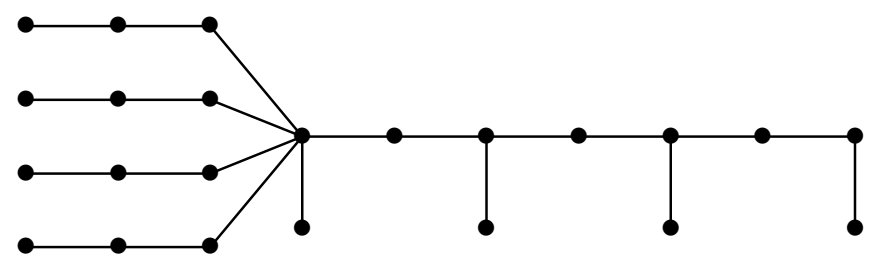

Figure 1: An extremal tree with maximum degree 6 and diameter 10.

When $k=3$, the lower bound $3(k+D / 2)-10$ on $n$ equals the upper bound $3 D / 2-1$, so the construction is finished. Henceforth we assume $k>3$.

To increase the number of vertices, note that except for $v$, the neighbors of leaves have degree 3 or 2. Augmenting at neighbors of endpoints of longest paths would increase the diameter (see Figure 1), but we can augment at neighbors of other leaves, increasing the number of vertices by 3 with each augmentation. We can continue such augmentations until every neighbor of a leaf that is not an endpoint of a longest path has degree $k$.

To count the vertices, it is helpful to grow such a tree in another way. When $D / 2$ is odd, let $\ell=(D-2) / 4$. Again start with $P_{5}$ and let $v$ be the neighbor of a leaf; $v$ has degree 2. Augmenting $k-2$ times at $v$ completes level 1 ; now $v$ has degree $k$, and the $k-1$ vertices at distance 2 from $v$ are neighbors of leaves (see the leftmost part of Figure 1). For $i \geq 1$, augment $k-2$ times at the neighbor of each leaf introduced when forming level $i$, until $\ell$ levels are complete. After level 1 , the diameter is 6 . Each successive level adds 4 to the diameter, so the resulting diameter is $6+4(\ell-1)$, which equals $D$. Counting the initial $P_{5}$ as a (degenerate) augmentation of $P_{2}$ to start level 1 , reaching the largest possible tree takes $\sum_{i=1}^{\ell}(k-1)(k-2)^{i-1}$ augmentations, so that tree has $2+3(k-1) \frac{(k-2)^{\ell}-1}{k-3}$ vertices. The augmentations can be reordered to begin with the initial example above having $3(k+D / 2)-10$ vertices, so all intermediate values congruent to 2 modulo 3 are attainable.

When $D / 2$ is even, instead build $\ell$ levels equally from the neighbors of both leaves of $P_{5}$, where $\ell=(D-4) / 4$. There are $k-2$ augmentations at each of them to complete level 1 , reaching diameter 8 . Augment $k-2$ times at the neighbor of each new leaf to complete level 2 , and so on through level $\ell$. Starting with the initial $P_{5}$ and counting the subtree from the 
neighbors of both leaves, the resulting maximum number of vertices is $5+6(k-2) \frac{(k-2)^{\ell}-1}{k-3}$.

When $n \not \equiv 2 \bmod 3$, simply use the construction $T^{\prime}$ for the next smaller number of vertices congruent to 2 modulo 3 and duplicate a leaf at the end of a longest path. Lemma 2.1 again yields the desired lower bound, and the Lampert-Slater bound requires equality.

As noted in the introduction, when $n \equiv 2 \bmod 3$, the family $\mathcal{T}$ is the family of all $n$ vertex graphs achieving the maximum value of the aquisition number, $(n+1) / 3$ (except also $C_{5}$ when $\left.n=5\right)$. The proof that this is the complete extremal family will appear in [6].

Theorem 2.2 solves the extremal problem for $n$-vertex trees with diameter $D$ whenever $6 \leq D \leq \frac{2}{3}(n+1)$, the upper bound being imposed by requiring $k \geq 3$ in the construction. When $D$ is larger, fewer vertices are available outside a longest path, and hence we cannot have as many of the leaves that by Lemma 2.1 force up the acquisition number. At $D=n-1$, the graph reduces to $P_{n}$. More generally, when $D>\frac{2}{3}(n+1)$, the maximum of the acquisition number is achieved by a special caterpillar. We begin by bounding the value on caterpillars.

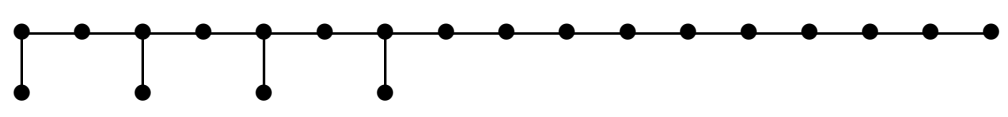

Figure 2: An extremal tree with high diameter.

Proposition 2.4. If $T$ is an $n$-vertex caterpillar with diameter $D$, then $a_{t}(T) \leq\left\lceil\frac{2 n-1-D}{4}\right\rceil$. Proof. We will use induction on $D$. If $D \leq 3$, then $T$ is $P_{1}, P_{2}$, a star, or a double-star. In each case $a_{t}(T)=1 \leq\left\lceil\frac{2 n-1-D}{4}\right\rceil$. Let $T$ be a caterpillar with diameter $D$, where $D \geq 4$, and let $P$ be a longest path in $T$. Let the vertices of $P$ be $v_{1}, \ldots, v_{D+1}$, indexed by their order along the path $P$. Let $C$ and $C^{\prime}$ be the components of $T-v_{3} v_{4}$ containing $v_{3}$ and $v_{4}$, respectively. Observe that $C$ is a caterpillar with diameter at most 3 and that $C^{\prime}$ is a caterpillar with diameter at most $D-2$ and with at most $n-3$ vertices. Applying the induction hypothesis yields $a_{t}(T) \leq a_{t}(C)+a_{t}\left(C^{\prime}\right) \leq 1+\left\lceil\frac{2(n-3)-1-(D-2)}{4}\right\rceil=\left\lceil\frac{2 n-1-D}{4}\right\rceil$

Theorem 2.5. For $\frac{2}{3}(n+1)<D \leq n-1$, the maximum of $a_{t}(T)$ when $T$ is an $n$-vertex tree with diameter $D$ is $\left\lceil\frac{2 n-1-D}{4}\right\rceil$.

Proof. We first provide a construction to prove sharpness of the upper bound. When $D=$ $n-1$, the tree is a path and has the desired acquisition number, so assume $D<n-1$. Begin by letting $T^{\prime}$ be the tree produced in Corollary 2.3 for maximum degree 3 and diameter $2(n-D)$ (when $D=n-2$ this initial subtree is $\left.P_{5}\right)$. Note that $T^{\prime}$ has $3(n-D)-1$ vertices and $n-D$ leaves. From the neighbor of an endpoint of a longest path in $T^{\prime}$, grow a path $P$ of length $3 D-2 n+1$ through $3 D-2 n+1$ new vertices; this completes $T$ with $n$ vertices and diameter $D$ (see Figure 2). 
By Lemma 2.1, weight from two leaves of $T^{\prime}$ cannot reach a common vertex, and they cannot combine with weight from $P$ that starts farther along $P$ than the second vertex. Since $a_{t}\left(P_{r}\right)=\lceil r / 4\rceil$ (Example 1.2), we have $a_{t}(T) \geq n-D+a_{t}\left(P_{3 D-2 n-1}\right)=\left\lceil\frac{2 n-D-1}{4}\right\rceil$. Equality holds, since the neighbors of leaves in $T^{\prime}$ can each acquire weight 3 , with the endpoint of $P$ also acquiring the weight from the second new vertex along $P$.

Now we prove the upper bound. For fixed $D$, we use induction on $n$. If $n=D+1$, then $T=P_{n}$ and $a_{t}(T)=\left\lceil\frac{2 n-1-D}{4}\right\rceil$. For a larger tree $T$, let $P$ be a longest path in $T$. If $T$ is a caterpillar, then Proposition 2.4 applies. Otherwise, there is a nontrivial component $C$ of $T-V(P)$. Let $s=|V(C)|$. Note that $a_{t}(T) \leq a_{t}(C)+a_{t}(T-V(C))$. The tree $T-V(C)$ has diameter $D$ and $n-s$ vertices. By the induction hypothesis, $a_{t}(T-V(C)) \leq\left\lceil\frac{2(n-s)-1-D}{4}\right\rceil$. The bound of Lampert and Slater gives $a_{t}(C) \leq\left\lfloor\frac{s+1}{3}\right\rfloor$. We compute

$$
\begin{aligned}
\left\lfloor\frac{s+1}{3}\right\rfloor+\left\lceil\frac{2 n-2 s-1-D}{4}\right\rceil & =\left\lceil\frac{s-1}{3}\right\rceil+\left\lceil\frac{2 n-2 s-1-D}{4}\right\rceil \\
& \leq\left\lceil\frac{2 n-1-D}{4}+\frac{2 s-3 s-2}{6}\right\rceil \leq\left\lceil\frac{2 n-1-D}{4}\right\rceil
\end{aligned}
$$

\section{Trees of Small Diameter}

Since trees with diameter 2 or 3 have total acquisition number 1, Theorem 2.2 and Proposition 2.5 leave only the extremal problems for diameter 4 and 5. These are settled in Theorems 3.3 and 3.4. We next define an acquisition protocol used in the proof of Theorem 3.3 and also in Section 5. It moves the maximum amount of weight to the central vertex in a tree of diameter 4.

Definition 3.1. Let $T$ be a tree with diameter 4 , and let $u$ be the center of $T$. Let $v_{1}, \ldots, v_{k}$ be the neighbors of $u$ labeled in nondecreasing order of degree (as in Figure 3). Define the $u$-greedy protocol, denoted $\mathcal{A}(u)$, as follows. Let $w_{i}$ denote the weight on $u$ at the beginning of step $i$; initially, $w_{1}=1$. In step $i$, move weight $\min \left\{w_{i}, d\left(v_{i}\right)\right\}-1$ from leaf neighbors of $v_{i}$ to $v_{i}$; the weight on $v_{i}$ is now $\min \left\{w_{i}, d\left(v_{i}\right)\right\}$. Complete step $i$ by transferring all weight on $v_{i}$ to $u$. Thus $w_{i+1}=w_{i}+\min \left\{w_{i}, d\left(v_{i}\right)\right\}$.

A closer look at the $u$-greedy protocol yields an upper bound for $a_{t}(T)$.

Lemma 3.2. Let $T$ be a tree with diameter 4 , let $u$ be the center of $T$, and run the $u$ greedy protocol $\mathcal{A}(u)$ on $T$. No weight remains on $N_{T}(u)$. Also, if $r$ is the degree of the highest-indexed neighbor of $u$ having leaf neighbors with positive weight after $\mathcal{A}(u)$, then at most $\lceil\lg r\rceil$ neighbors of $u$ have leaf neighbors with positive weight after $\mathcal{A}(u)$. Consequently, $a_{t}(T) \leq r\lceil\lg r\rceil$. 
Proof. By construction, no weight remains on $N(u)$. Now, let $S$ be the set of neighbors of $u$ having leaf neighbors with positive weight after $\mathcal{A}(u)$. Let $m=\max \left\{i: v_{i} \in S\right\}$, so $d\left(v_{m}\right)=r$. If $v_{i} \in S$, then $d\left(v_{i}\right)>w_{i}$. Therefore, the weight at $u$ doubles during step $i$ for each $i \in S$, and $r>w_{m}$. Hence the weight on $u$ has doubled at most $\lg (r-1)$ times by step $m$, so $|S| \leq 1+\lfloor\lg (r-1)\rfloor=\lceil\lg r\rceil$. Since each vertex in $S$ has at most $r-1$ leaf neighbors, $a_{t}(T) \leq r\lceil\lg r\rceil$.

Our upper bound for trees with diameter 4 uses the $u$-greedy protocol when the degree of the central vertex is large and none of its neighbors have large degree.

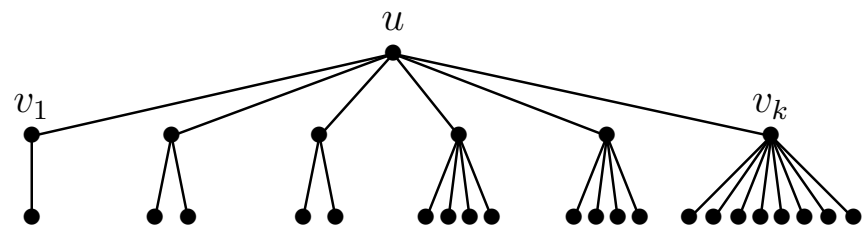

Figure 3: Vertex labeling for trees of diameter 4

Theorem 3.3. Let $T$ be an $n$-vertex tree. If $T$ has diameter 4 , then $a_{t}(T) \leq \sqrt{n \lg n}$. If $T$ has diameter 5 , then $a_{t}(T) \leq \sqrt{2 n \lg n}$.

Proof. First consider diameter 4; we prove the bound by induction on $n$. Let $u$ be the central vertex of $T$, and label its neighbors $v_{1}, \ldots, v_{k}$ in nondecreasing order of degree, as in Figure 3.

If $k \leq \sqrt{n \lg n}$, then it suffices to let the neighbors of $u$ absorb all the weight. If $k>$ $\sqrt{n \lg n}$, then $k \geq n / 2$ if $n \leq 16$. We will show in Lemma 4.3 that $a_{t}(G)=1$ when $G$ has a vertex of degree at least $|V(G)| / 2$ whose neighborhood is a dominating set. Hence we may assume that $k>\sqrt{n \lg n}$ and $n>16$.

If $d\left(v_{k}\right) \geq \sqrt{n}$, then we let $v_{k}$ acquire the weight on its leaf neighbors and apply the induction hypothesis to the tree obtained by deleting $v_{k}$ and its leaf neighbors. Thus $a_{t}(T) \leq$ $1+\sqrt{(n-\sqrt{n}) \lg n}$. Note that $1+\sqrt{A-B} \leq \sqrt{A}$ if and only if $B \geq 2 \sqrt{A}-1$. Since $\sqrt{n} \lg n \geq 2 \sqrt{n \lg n}$ when $n \geq 16$, this case is complete.

Hence we may assume that $k>\sqrt{n \lg n}$, that $d\left(v_{k}\right)<\sqrt{n}$, and that $n>16$. Let $S$ be the set of neighbors of $u$ having leaf neighbors with positive weight after running the $u$-greedy protocol $\mathcal{A}(u)$. Let $m=\max \left\{i: v_{i} \in S\right\}$. By Lemma 3.2, $a_{t}(T) \leq d\left(v_{m}\right)\left\lceil\lg d\left(v_{m}\right)\right\rceil$.

We will prove that $d\left(v_{m}\right)<2 n / k-1$. Given this, and using $k \geq\lceil\sqrt{n \lg n}$,

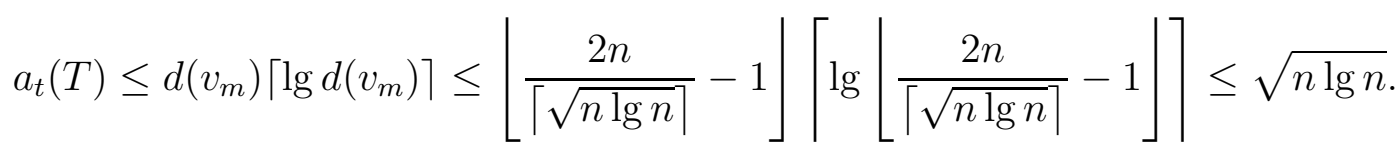


For $n>2^{16}$, the last bounds follows, since in this case

$$
\left\lfloor\frac{2 n}{\lceil\sqrt{n \lg n}\rceil}-1\right\rfloor\left\lceil\lg \left\lfloor\frac{2 n}{\lceil\sqrt{n \lg n}\rceil}-1\right\rfloor<2 \sqrt{\frac{n}{\lg n}}\left[\lg \left(2 \sqrt{\frac{n}{\lg n}}\right)+1\right] \leq \sqrt{n \lg n} .\right.
$$

For $n \leq 2^{16}$, the desired inequality holds by explicit computation.

To prove $d\left(v_{m}\right)<2 n / k-1$, we first argue that $m<k / 2$. Since $u$ acquires weight with each step, $m \leq w_{m}$. Since $m \in S$, we have $w_{m}<d\left(v_{m}\right)$. We are in the case where $d\left(v_{k}\right)<\sqrt{n}$. Finally, $k>\sqrt{n \lg n}$ yields $\sqrt{n}<k / 2$ when $n>16$. Thus

$$
m \leq w_{m}<d\left(v_{m}\right) \leq d\left(v_{k}\right)<\sqrt{n}<k / 2 .
$$

Since the $k-m+1$ neighbors of $u$ in $\left\{v_{m}, \ldots, v_{k}\right\}$ each have at least $d\left(v_{m}\right)-1$ leaf neighbors, and at least $m$ vertices are outside the subtrees rooted at those neighbors, $d\left(v_{m}\right)<$ $\frac{n-m}{k-m}$. With $m<k / 2$, we have $d\left(v_{m}\right)<2 n / k-1$. This completes the proof for diameter 4 .

When $T$ has diameter 5 , deleting the central edge of $T$ leaves two subtrees with diameter at most 4 , using $p$ and $n-p$ vertices, for some $p$. Applying the bound for diameter 4 and the concavity of that bound yields

$$
a_{t}(T) \leq \sqrt{p \lg p}+\sqrt{(n-p) \lg (n-p)} \leq 2 \sqrt{(n / 2) \lg (n / 2)}<\sqrt{2 n \lg n},
$$

which completes the proof.

The bound of Theorem 3.3 for diameter 4 is within a factor of $\sqrt{2}$ of being sharp.

Theorem 3.4. For sufficiently large $n$, there is an $n$-vertex tree $T_{n}$ with diameter 4 and $a_{t}\left(T_{n}\right) \geq(1-o(1)) \sqrt{\frac{1}{2} n \lg n}$.

Proof. Let $T_{n}$ have central vertex $u$, and let $r=\sqrt{2 n / \lg n}$. Let $u$ have degree $k$, where $k=\left\lfloor\frac{n-1}{\lfloor r\rfloor+1}\right\rfloor$. Let the neighbors $v_{1}, \ldots, v_{k}$ of $u$ all have degree $\lceil r\rceil$ or $\lfloor r\rfloor$. Note that $k=$ $(1-o(1)) \sqrt{\frac{1}{2} n \lg n}$. We prove that $a_{t}\left(T_{n}\right) \geq k$.

Let $\mathcal{A}$ be an optimal acquisition protocol on $T_{n}$, and let $q$ be the number of neighbors of $u$ that transfer weight to $u$. Without loss of generality, we can assume that weight moves from $v_{1}, \ldots, v_{q}$ to $u$ in order. To minimize the residual set, $\mathcal{A}$ transfers the weight from all the leaf neighbors of $v_{i}$ to $v_{i}$ for $q<i \leq k$.

Let $T^{\prime}$ be the subtree obtained by deleting $v_{q+1}, \ldots, v_{k}$ and their leaf neighbors. Since weight moves from $v_{i}$ to $u$ for $1 \leq i \leq q$, the residual set in $T^{\prime}$ consists of $u$ and some of the leaves. To minimize the number of leaves in the residual set, on $T^{\prime}$ the weight of $u$ should be maximized, and hence $\mathcal{A}$ should run the $u$-greedy protocol on $T^{\prime}$.

If $q<\lg r$, then weight remains on a leaf neighbor of each $v_{i}$ with $1 \leq i \leq q$ and also on $v_{q+1}, \ldots, v_{k}$, so weight remains on at least $k$ vertices, and $a_{t}\left(T_{n}\right) \geq k=(1-o(1)) \sqrt{\frac{1}{2} n \lg n}$. 
If $q>\lg r$, then because each neighbor has degree at least $\lfloor r\rfloor$, the $u$-greedy protocol on $T^{\prime}$ puts weight at least $\lceil r\rceil$ on $u$. Now $u$ is able to acquire all of the weight from each $v_{i}$ and its leaf neighbors when $i>q$, so it is not optimal to leave any weight there. Given that $\mathcal{A}$ is optimal, we conclude that $q=k$ when $q>\lg r$. Since the moved weight will double with each iteration for the first $\lfloor\lg r\rfloor$ iterations, the number of vertices stranded is at least $\sum_{i=1}^{\lfloor\lg r\rfloor}\left(r-\left(2^{i-1}-1\right)\right)$. We compute

$$
\begin{aligned}
a_{t}\left(T_{n}\right) & \geq r\lfloor\lg r\rfloor-\sum_{i=1}^{\lfloor\lg r\rfloor}\left(2^{i-1}-1\right)=(1-o(1))(r \lg r) \\
& =(1-o(1)) \sqrt{\frac{2 n}{\lg n}} \lg \sqrt{\frac{2 n}{\lg n}}=(1-o(1)) \frac{1}{2} \sqrt{\frac{2 n}{\lg n}} \lg n=(1-o(1)) \sqrt{\frac{n \lg n}{2}} .
\end{aligned}
$$

Slater and Wang [11] proved that the problem of determining $a_{t}(G)$ for general graphs is NP-complete. In fact, it is NP-complete even to test whether $a_{t}(G)=1$. They asked whether the same statements are true when the problem is restricted to trees. We partly answer this by providing for any fixed $k$ a polynomial-time algorithm to determine whether $a_{t}(T) \leq k$. We start by characterizing trees with acquisition number 1 .

A rooted tree $(T, r)$ consists of a tree $T$ and a distinguished vertex $r \in V(T)$. A rooted acquisition tree is a rooted tree $(T, r)$ such that some acquisition protocol transfers all the weight in $T$ to $r$.

Lemma 3.5. A rooted tree $(T, r)$ is a rooted acquisition tree if and only if 1) $|V(T)|=1$, or

2) $T$ has an edge $\mathrm{rr}^{\prime}$ whose deletion leaves rooted acquisition trees rooted at $r$ and $r^{\prime}$, such that the component containing $r^{\prime}$ is no bigger than the component containing $r$.

Proof. For necessity, let $r r^{\prime}$ be the last edge used in an acquisition protocol that moves all weight to $r$. For sufficiency, use $r r^{\prime}$ after such protocols in the two subtrees.

The recursive characterization of rooted acquisition trees in Lemma 3.5 is just the definition of union trees, a class of trees used as a data structure in computer science. Thus

$(T, r)$ is a rooted acquisition tree if and only if it is a union tree.

Cai [3] characterized union trees and gave a $O\left(n^{2}\right)$-time algorithm to recognize them. We use it in our algorithm for testing $a_{t}(T) \leq k$.

Theorem 3.6. For each positive integer $k$, there is an $O\left(|V(T)|^{k+2}\right)$-time algorithm for testing $a_{t}(T) \leq k$ when $T$ is a tree. 
Proof. The edges along which weight moves to an element of the residual set form a subtree, and these subtrees are disjoint. Thus $a_{t}(T) \leq k$ if and only if there are $k$ disjoint rooted acquisition trees in $T$ that together span $V(T)$. Deletion of an edge from a tree increases the number of components by 1 . Thus $k$ disjoint subtrees spanning $V(T)$ are obtained by deleting $k-1$ edges of $T$.

For all $B \subseteq E(T)$ with $|B|=k-1$, let $T_{1}, \ldots, T_{k}$ be the components of $T-B$. For each vertex $r \in V\left(T_{i}\right)$, use Cai's algorithm to test whether $\left(T_{i}, r\right)$ is a rooted acquisition tree. Conclude $a_{t}(T) \leq k$ if and only if, for some $B$, each component of $T-B$ is a rooted acquisition tree rooted at one of its vertices.

There are $O\left(n^{k-1}\right)$ choices for $B$. Given $B$, testing whether a vertex of $T$ is a suitable root for its component takes at most quadratic time, using Cai's algorithm, and we need only test a linear number of roots. Thus our algorithm runs in $O\left(n^{k+2}\right)$ time.

\section{Sufficient Conditions for $a_{t}(G)=1$}

Since recognizing graphs with acquisition number 1 is NP-hard, it is natural to seek sufficient conditions for that property; such conditions are the goal of this section. A clique in a graph is a set of pairwise adjacent vertices; a dominating clique is a clique that is a dominating set.

Proposition 4.1. If a graph $G$ has a dominating clique, then $a_{t}(G)=1$.

Proof. When $K$ is a dominating clique, we can move all weight from $V(G)-K$ onto $K$ and then consolidate all weight onto a single vertex using edges within $K$.

An $H$-free graph is a graph not having $H$ as an induced subgraph. Bacsó and Tuza [1] showed that every connected graph that is both $P_{5}$-free and $C_{5}$-free has a dominating clique. Thus Proposition 4.1 has a corollary.

Corollary 4.2. If a graph $G$ is connected, $P_{5}$-free, and $C_{5}$-free, then $a_{t}(G)=1$.

Our next objective is to show that if $G \neq C_{5}$, then $a_{t}(G)=1$ or $a_{t}(\bar{G})=1$. We first prove that $a_{t}(G)=1$ if the neighborhood of a vertex with sufficiently high degree is a dominating set. We then show that if $G \neq C_{5}$ and $G$ is $(|V(G)|-1) / 2$-regular, then $a_{t}(G)=1$. Together with Proposition 4.1, these results will complete the proof. Let $N_{G}(v)$ or $N(v)$ denote the set of neighbors of vertex $v$, and let $N[v]=N(v) \cup\{v\}$.

Lemma 4.3. If $G$ is an $n$-vertex graph having a vertex $v$ such that $d(v) \geq n / 2$ and $N(v)$ is a dominating set, then $a_{t}(G)=1$. 
Proof. Since $N(v)$ is a dominating set, we can first move all weight from $V(G)-N[v]$ onto $N(v)$. Let $\alpha$ be the maximum of the weights on vertices of $N(v)$ at this point. Note that $\alpha-1$ units of weight came to one vertex of $N(v)$ from vertices among the $n-1-d(v)$ vertices of $V(G)-N[v]$. Since $d(v) \geq n / 2$, at most $n / 2-\alpha$ units of weight have been moved from $V(G)-N[v]$ to other vertices of $N(v)$, and hence there remain at least $\alpha-1$ vertices in $N(v)$ with weight 1 . Moving the weight from each neighbor of $v$ with weight 1 to $v$ gives $v$ weight at least $\alpha$, after which all remaining weight can be moved to $v$.

Lemma 4.4. If $G$ is an n-vertex, $(n-1) / 2$-regular graph other than $C_{5}$, then $a_{t}(G)=1$.

Proof. Since $C_{5}$ is excluded, we may assume $n \geq 7$. Choose $v \in V(G)$. Since any two nonadjacent vertices have a common neighbor, $N(v)$ is a dominating set. Let $x$ be a vertex of $N(v)$ with the most neighbors outside $N[v]$. Let $R=V(G)-N[v]$.

If $|N(x) \cap R| \geq 2$, then move all weight from $N(x)-N[v]$ onto $x$; let its weight now be $\alpha$. Move the rest of the weight from $R$ onto $N(v)-\{x\}$. Choose $y \in N(v)-\{x\}$ having maximum weight at this point; let its weight be $\beta$.

We have moved weight $\alpha+\beta-2$ from $R$ onto $\{x, y\}$. Hence weight $(n-1) / 2-(\alpha+\beta-2)$ was moved onto the $(n-1) / 2-2$ vertices of $N(v)-\{x, y\}$, leaving at least $\alpha+\beta-4$ vertices there with weight 1 . Let $v$ acquire the weight on those vertices, reaching weight $\alpha+\beta-3$. Since $\alpha \geq 3$, now $v$ has weight at least $\beta$ and can acquire the weight from all vertices of $N(v)-\{x\}$, after which weight remains on only $v$ and $x$, which are adjacent.

The remaining case is $\alpha=2$. This requires each vertex of $N(v)$ to have at most one neighbor in $R$, so each vertex of $R$ has exactly one neighbor in $N[v]$ and $(n-3) / 2$ neighbors in $R$. Thus $R$ is a clique, and each vertex of $N(v)$ is adjacent to all but one vertex of $N(v)$. Since $d(v) \geq 3$, there is an edge within $N(v)$. Each such edge is a dominating clique for the subgraph induced by $N[v]$, and we can acquire all weight from that subgraph onto a vertex $z$ of $N(v)$. Since $R$ is a clique, we can move all its weight to the neighbor of $z$ in $R$. Now all weight from $G$ rests on two neighboring vertices.

Theorem 4.5. If $G$ is a graph and $G \neq C_{5}$, then $a_{t}(G)$ or $a_{t}(\bar{G})$ equals 1 .

Proof. If $G$ has diameter at least 3 , then any pair of vertices $x$ and $y$ satisfying $d_{G}(x, y) \geq 3$ form a dominating clique in $\bar{G}$, so $a_{t}(\bar{G})=1$ by Proposition 4.1 . By symmetry, we may assume that $G$ and $\bar{G}$ both have diameter 2 .

Let $n=|V(G)|$. If $G$ has a vertex $v$ of degree at least $n / 2$, then $N(v)$ dominates $G$ (since $G$ has diameter 2), so Lemma 4.3 implies $a_{t}(G)=1$. We may assume, therefore, that $\Delta(G)$ and $\Delta(\bar{G})$ (by symmetry) are at most $(n-1) / 2$. It follows that $G$ is $(n-1) / 2$-regular, and Lemma 4.4 yields $a_{t}(G)=1$. 
Ore's Theorem [8] states that if any two nonadjacent vertices in an $n$-vertex graph have degree sum at least $n$, then the graph has a spanning cycle. A similar condition guarantees total acquisition number 1 .

Theorem 4.6. Let $G$ be an n-vertex graph other than $C_{5}$. If $d(u)+d(v) \geq n-1$ whenever $u$ and $v$ are nonadjacent vertices in $G$, then $a_{t}(G)=1$.

Proof. The hypothesis guarantees that any two nonadjacent vertices have a common neighbor, so every vertex neighborhood is a dominating set. If some vertex has degree at least $n / 2$, then Lemma 4.3 applies. Otherwise, $G$ is $(n-1) / 2$-regular and Lemma 4.4 applies.

Theorem 4.6 implies that $a_{t}(G)=1$ when $\delta(G) \geq(|V(G)|-1) / 2$. This threshold is sharp, since $2 K_{n / 2}$ has minimum degree $(n-2) / 2$ and acquisition number 2 (when $n$ is even).

\section{Diameter 2}

Intuitively, graphs with diameter 2 should have small acquisition numbers, since it is easier to move weight smaller distances. Because every graph with diameter 2 has a spanning tree with diameter at most 4 , it is natural to apply Lemma 3.2 to these graphs. Lemma 3.2 implies that if $u$ is a vertex in a graph $G$ with diameter 2 , and $d=\max _{v \in N(u)}(N(v)-N[u])$, then $a_{t}(G) \leq d\lceil\lg (d)\rceil$. Our first goal in this section is to prove a better bound that is nearly logarithmic in the number of vertices.

Nevertheless, we conjecture a much stronger upper bound.

Conjecture 5.1. There is an absolute constant $c$ such that $a_{t}(G) \leq c$ whenever $G$ has diameter 2 .

In fact, we know of no graph with diameter 2 having acquisition number more than 2 . For example, the only nontrivial Cartesian products with diameter 2 are Cartesian products of two complete graphs, and $a_{t}\left(K_{r} \square K_{s}\right)=1$. Also, $a_{t}\left(C_{5} \otimes C_{5}\right)=1$ (see Section 7). Although we cannot prove Conjecture 5.1, we will prove the constant bound for graphs having diameter 2 and no 4-cycle. With four exceptions, these graphs all have acquisition number 1.

Of course, many graphs with diameter 2 have 4-cycles, which brings us back to the general bound for graphs with diameter 2. To prove this bound, we will use another protocol in conjunction with the $u$-greedy protocol. Roughly speaking, it provides a better bound when the degree of the center is large and the number of vertices at distance 2 is small.

Lemma 5.2. Let $T$ be a tree with diameter 4 and let $u$ be the center of $T$. Let $d=d_{T}(u)$, and let $R=V(T)-N[u]$. Suppose $d \geq 256$. If $|R| \leq d\lceil\lg d\rceil$, then $a_{t}(T) \leq 10 \lg d \lg \lg d$. Also, under some protocol achieving this bound, $u$ acquires weight at least $d-4\lceil\lg d\rceil$. 
Proof. Let $N_{1}$ be the set of neighbors of $u$ with degree less than $4 \lg d$, let $N_{2}$ be the set of neighbors of $u$ with degree at least $4 \lg d$ and less than $d / 4$, and let $N_{3}$ be the set of neighbors of $u$ with degree at least $d / 4$ (See Figure 4). For $i \in\{1,2\}$, let $T_{i}$ be the subtree of $T$ with vertex set $\bigcup_{x \in N_{i}} N[x]$. We will apply the $u$-greedy protocol to $T_{1}$ and then show that this gives $u$ enough weight to acquire all of the weight in $T_{2}$.

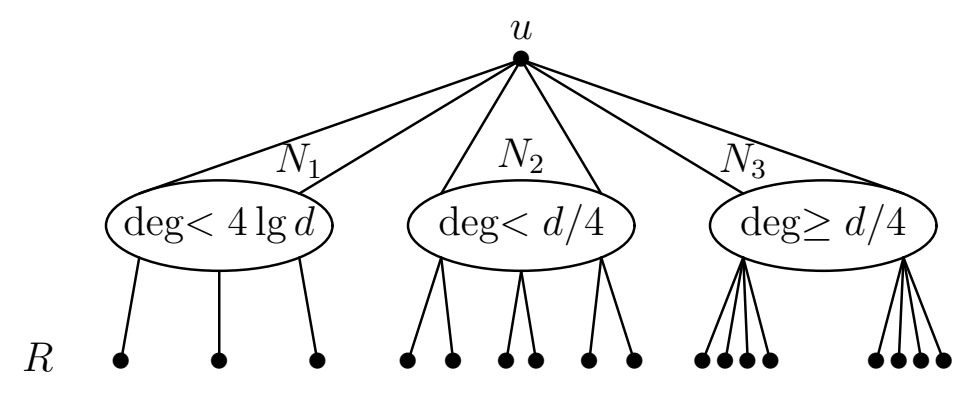

Figure 4: The partition of $N(u)$ for Lemma 5.2.

We have $\left|N_{1}\right| \geq d / 4$, since otherwise $|R|>\frac{3 d}{4}(4 \lg d-1)>d\lceil\lg d\rceil \geq|R|$. By Lemma 3.2 there is a total acquisition protocol on $T_{1}$ that moves weight from all but $4 \lg d\lceil\lg (4 \lg d)\rceil$ vertices in $T_{1}$ to $u$. Note that $4 \lg d\lceil\lg (4 \lg d)\rceil \leq(4 \lg d)(3+\lg \lg d) \leq 8 \lg d \lg \lg d$, since $d \geq 256$. Also, $u$ now has weight at least $\left|N_{1}\right|$. Since $\left|N_{1}\right| \geq d(x)$ for $x \in N_{2}$, we can transfer all the weight in $T_{2}$ to $u$. Finally, transfer all weight from $\bigcup_{x \in N_{3}} N(x)-u$ to $N_{3}$.

Since $|R| \leq d\lceil\lg d\rceil$, we have $\left|N_{3}\right| \leq|R| /(d / 4) \leq 4+4 \lg d$. Therefore,

$$
a_{t}(T) \leq 1+8 \lg d \lg \lg d+\left|N_{3}\right| \leq 10 \lg d \lg \lg d,
$$

since $d \geq 256$. Also, the weight on $u$ is at least $\left|N_{1}\right|+\left|N_{2}\right|$, which is at least $d-4\lceil\lg d\rceil$.

We now prove our bound for graphs with diameter 2. The proof starts by applying the $u$-greedy protocol to a subgraph of $G$, where $u$ is a vertex of maximum degree. We then apply Lemma 5.2 to a subtree where the leaves are the vertices with weight 1 after the application of the $u$-greedy protocol. Combining the two protocols yields a much stronger bound.

Theorem 5.3. If $G$ is an $n$-vertex graph with diameter 2 , then $a_{t}(G) \leq 32 \lg n \lg \lg n$.

Proof. For the main case, we will need $n>256^{2}$. For $n \leq 256^{2}$, we apply Theorem 3.3. We obtain a spanning tree of diameter 4 in $G$ showing that $a_{t}(G) \leq \sqrt{n \lg n}$, and therefore

$$
a_{t}(G) \leq 16 \lg n \lg \lg n .
$$

Henceforth we assume that $n>256^{2}$. Let $d=\Delta(G)$, and let $u$ be a vertex of maximum degree. Since $G$ has diameter 2 , we have $d \geq \sqrt{n-1} \geq 256$. Let $N(u)=\left\{v_{1}, \ldots, v_{d}\right\}$ and 
let $R=V(G)-N[u]$. Among all vertices in $N(u)$, let $v$ be one with the most neighbors in $R$. Let $r=|N(v) \cap R|$. If $r<256$, then $G$ has a spanning tree $T$ with diameter 4 centered at $u$ such that $\max _{x \in N_{T}(u)} d_{T}(x) \leq 256$. Lemma 3.2 then yields

$$
a_{t}(G) \leq 256\lceil\lg 256\rceil=32 \lg 256^{2} \lg \lg 256^{2} \leq 32 \lg n \lg \lg n .
$$

Thus we may assume $r \geq 256$. Let $S=N(u) \cap N(v)$ and $M=N(u)-N[v]$. Let $W$ be the subset of $R$ consisting of vertices with no neighbor in $S \cup\{v\}$ (See Figure 5).

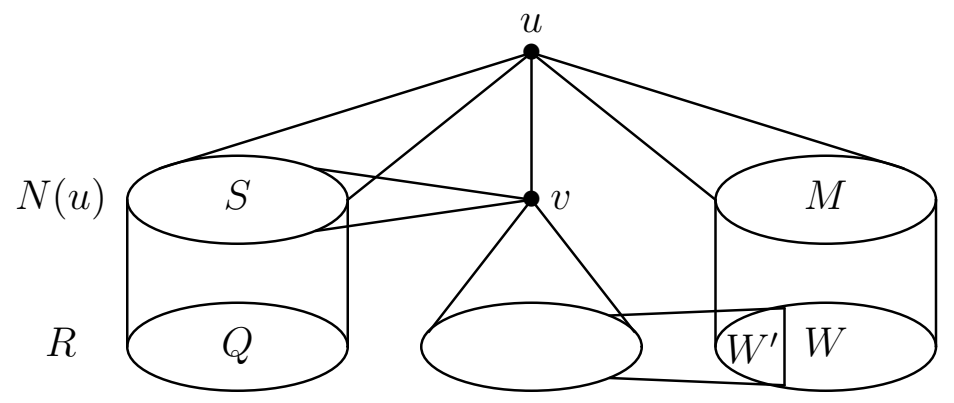

Figure 5: The structure of the diameter 2 graph $G$ in Theorem 5.3.

Since all of $W$ is within distance 2 of $u$, every vertex of $W$ has a neighbor in $M$. Let $T$ be a spanning tree of diameter 4 with center $u$ in the subgraph of $G$ induced by $\{u\} \cup M \cup W$. Let $k=\max _{x \in M}\left|N_{T}(x) \cap W\right|-1$; note that $k<r$. Lemma 3.2 applied to the subtree yields a protocol on $T$ that leaves weight on fewer than $k\lceil\lg k\rceil$ vertices in $W$ and no weight on $M$. Let $W^{\prime}$ be the set of vertices in $W$ that retain positive weight; note that $\left|W^{\prime}\right|<k\lceil\lg k\rceil$.

Since all of $W^{\prime}$ is within distance 2 of $v$, vertices of $W^{\prime}$ have common neighbors with $v$, which can only be in $N(v) \cap R$. Let $H$ be the subgraph of $G$ induced by $W^{\prime} \cup\{v\} \cup(N(v) \cap R)$. Note that $d_{H}(v)=r \geq 256$; we will apply Lemma 5.2 to a suitable spanning tree of $H$. Since all of $H$ is within distance 2 of $v$, there is in $H$ a spanning tree $T^{\prime}$ of diameter 4 with $v$ as its center, such that $d_{T^{\prime}}(v)=r>k$ and $\left|W^{\prime}\right|<k\lceil\lg k\rceil$. Because $k<d_{H}(v)=r$, Lemma 5.2 implies $a_{t}(H) \leq 10 \lg r \lg \lg r$, and there is a protocol achieving this bound such that the weight at $v$ is $m$, where $m \geq r-4\lceil\lg r\rceil$.

Let $Q=R-W-N(v)$. The only remaining vertices outside of $V(H) \cup\{u\}$ with positive weight are in $S \cup Q$. Among vertices in $S$, let $y$ be one with the most neighbors in $Q$. If $m>|Q \cap N(y)|$, then the weight on $v$ is greater than $\left|Q \cap N\left(v_{i}\right)\right|$ for all $v_{i} \in S$, and we can transfer all weight from $S \cup Q$ to $v$. By then transferring weight along the edge $u v$, the weight is consolidated on at most $1+10 \lg d \lg \lg d$ vertices.

If $m \leq|Q \cap N(y)|$, then transfer weight from $m-1$ vertices in $Q$ to $y$ and then move weight $m$ from $y$ to $v$. After this move, the weight on $v$ is $2 m$, and $2 m \geq 2 r-8\lceil\lg r\rceil$. Since $r \geq 256$, we have $2 r-8\lceil\lg r\rceil>r$, so the weight now on $v$ exceeds $\left|N\left(v_{i}\right) \cap Q\right|$ for each $v_{i} \in S$. In $N(y) \cap Q$, we left weight on at most $r-m+1$ vertices; note that $r-m+1 \leq 4\lceil\lg r\rceil+1$. 
Now all of the weight in $G$ lies on $u$, at most $10 \lg d \lg \lg d$ vertices in $H$, and at most $4\lceil\lg r\rceil+1$ vertices in $Q$. Since $r<d$,

$$
a_{t}(G) \leq 2+10 \lg d \lg \lg d+4\lceil\lg d\rceil \leq 12 \lg n \lg \lg n
$$

In all cases, $a_{t}(G) \leq 32 \lg n \lg \lg n$.

In the remainder of this section, we prove that $a_{t}(G)=1$ when $G$ has diameter 2 and no 4-cycle, with four exceptions. Note that graphs with diameter 2 have girth at most 5 . Very few have girth 5; these are called Moore graphs. They are regular and exist only for degrees 2, 3, 7, and possibly 57 [4]. The other possible graphs have triangles. The arguments for upper bounds will proceed by bringing the weight to a shortest cycle.

We begin by presenting the exceptional graphs; two with girth 5 and two with girth 3 . The two examples with triangles happen to be the polarity graphs of the projective planes of orders 2 and 3 ; for this reason we call them $F_{2}$ and $F_{3}$.

Lemma 5.4. Graphs with diameter 2, no 4-cycle, and acquisition number 2 include $C_{5}$, the Petersen graph, and the graphs $F_{2}$ and $F_{3}$ in Figure 6.

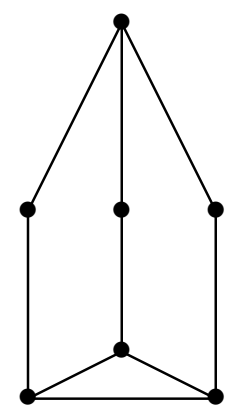

$F_{2}$

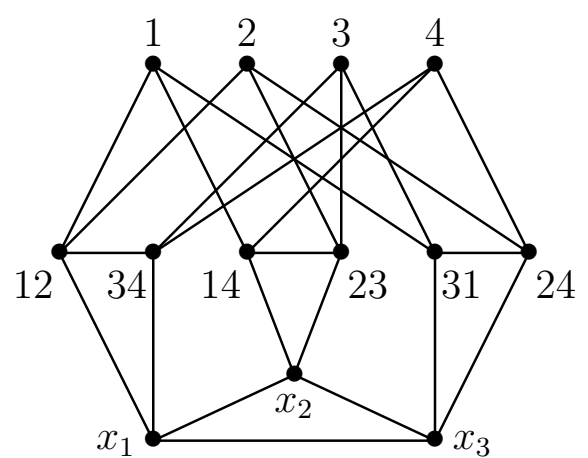

$F_{3}$

Figure 6: Graphs with diameter 2, no 4-cycle, and acquisition number 2.

Proof. Consider first $C_{5}$ and the Petersen graph. They have acquisition number at least 2, because that is required by $2^{\Delta(G)}<|V(G)|$. On the other hand, since the independence number of $C_{5}$ is 2 , the acquisition number of any graph having a 5-cycle whose vertices form a dominating set is at most 2 .

To prove the lower bound for $F_{2}$ and $F_{3}$, consider rooted acquisition trees. In a graph with maximum degree $D$, the weight of the root in a rooted acquisition tree before the final acquisition is at most $2^{D-1}$. Hence in a 7 -vertex graph with $D=3$, the final move combines weights 4 and 3 , while in a 13 -vertex graph with $D=4$, the final move combines weights 8 
and 5 or weights 7 and 6 . Hence both vertices in the final move have degree $D$ in the tree. However, in both cases every subgraph having adjacent vertices of degree $D$ has a triangle and cannot be a tree. With no rooted acquisition tree, the acquisition number cannot be 1 .

For the upper bound, the vertices of the graph on the left partition into two sets inducing graphs with acquisition number 1: a star and a triangle. For the graphs represented on the right, it suffices to show that all the weight except that on vertex 4 can be acquired to a single vertex. Simply aquire weight from $\{1,2,3,34,14,24\}$ to $\left\{12,23,31, x_{1}, x_{2}, x_{3}\right\}$ along a matching, after which there is weight 2 on every vertex of a 6 -vertex graph with a dominating triangle.

In order to prove that the other graphs with diameter 2 and no 4-cycle have acquisition number 1 , we need an old lemma that is not hard to prove.

Lemma 5.5. (Bondy, Erdős, and Fajtlowicz [2]) If a graph $G$ has diameter 2 and no 4cycle, and $x$ and $y$ are nonadjacent vertices with a common neighbor $u$, then $N(x)-N[u]$ and $N(y)-N[u]$ have the same size and are joined by matching in $G$.

Proof. Let $A=N(x)-N[u]$ and $B=N(y)-N[u]$. For $z \in A$, distance 2 to $y$ requires $z$ to have a neighbor in $B$, and avoiding 4-cycles prevents it from having more than 1 . Hence each vertex of $A$ has exactly one neighbor in $B$, and by symmetry each vertex of $B$ has exactly one neighbor in $A$.

In fact, the theorem proved in [2] is that if $G$ has diameter 2 and no 4-cycle, then one of the following holds: (a) $G$ has a dominating vertex, or (b) $G$ is a Moore graph (girth 5), or (c) $G$ is a polarity graph. Rather than use that conclusion and study the structure of these types of graphs separately using known properties, we instead develop common aspects directly from the hypothesis that allow us to bound the acquisition number when case (a) does not hold.

Lemma 5.6. Let $G$ be a graph with diameter 2 and no 4 -cycle, and let $x_{1}, \ldots, x_{g}$ (in order) induce a shortest cycle $C$ in $G$. Also let $R$ be the set of vertices having no neighbor in $C$, and let $X_{i}=N\left(x_{i}\right)-V(C)$. The sets $X_{1}, \ldots, X_{g}$ are disjoint and of equal size, $k$. Every vertex of $R$ has exactly one neighbor in each such set. For $z \in X_{i}$, the set $N(z) \cap R$ also has size $k$ if $g=3$, and it has size $k-1$ if $g=5$.

Proof. Since graphs with girth $g$ have diameter at least $(g-1) / 2$, we have $g \in\{3,5\}$. Avoiding 4-cycles (and 3-cycles when $g=5$ ) forces $X_{1}, \ldots, X_{g}$ to be disjoint. In order to stay within distance 2 of each vertex of $C$ (and avoid 4-cycles), each vertex of $R$ must have exactly one neighbor in each $X_{i}$.

If $G=C_{5}$, then $k=0$ and the statements about $z$ are vacuous. Otherwise, note that $N(z) \cap V(C)=\left\{x_{i}\right\}$ and view indices modulo $g$. Since $z$ and $x_{i+1}$ are nonadjacent and 
have $x_{i}$ as a common neighbor, Lemma 5.5 applies. Deleting $N\left[x_{i}\right]$ eliminates $x_{i-1}$ and all of $X_{i}$, leaving a matching that joins $N(z)-N\left[x_{i}\right]$ to $N\left(x_{i+1}\right)-N\left[x_{i}\right]$. If $g=3$, then these sets are $N(z) \cap R$ and $X_{i+1}$. If $g=5$, then reaching $x_{i+2}$ and $x_{i-2}$ in two steps requires $z$ to have one neighbor each in $X_{i+2}$ and $X_{i-2}$, and the second set is $X_{i+1} \cup\left\{x_{i+2}\right\}$; thus $|N(z) \cap R|=\left|X_{i+1}\right|-1$ in this case.

The same argument with $i-1$ in place of $i+1$ establishes the same relationship between $|N(z) \cap R|$ and $\left|X_{i-1}\right|$. Thus $\left|X_{i+1}\right|=\left|X_{i-1}\right|$. Calling this common value $k$, we also have $|N(z) \cap R|=k$ when $g=3$, and $|N(z) \cap R|=k-1$ when $g=5$.

Since $z$ and $i$ were arbitrary, the full claim follows.

We can now complete the analysis for graphs of diameter 2 with no 4-cycles. Obviously $a_{t}(G)=1$ when $G$ has a dominating vertex, so we consider only the remaining cases.

Theorem 5.7. Let $G$ be a graph with diameter 2 , no 4-cycle, and no dominating vertex. Define $g, C$, and $k$ and the various vertex subsets as in the statement of Lemma 5.6. If $k \geq 3$, then $a_{t}(G)=1$. Otherwise, $G$ is one of the four graphs in Lemma 5.4.

Proof. Each vertex of $R$ has one neighbor in $X_{i}$, and each vertex of $X_{i}$ has $k$ or $k-1$ neighbors in $R$, depending on $g$. Thus $|R|=k^{2}$ when $g=3$, and $|R|=k(k-1)$ when $g=5$. When $k \geq 3$, we will gather all weight onto $C$ (mostly at $x_{1}$ and $x_{2}$ ) and then to one vertex.

If $k=0$, then $G=C$. If $k=1$ and $g=3$, then $|R|=1$ and $G=F_{2}$. If $k=1$ and $g=5$, then $|R|=0$; since the one vertex of $X_{i}$ has neighbors in $X_{i+2}$ and $X_{i-2}$, we obtain the Petersen graph.

Before excluding $k=2$, we develop structure for $R$. Let $X_{1}=\left\{u_{1}, \ldots, u_{k}\right\}$ and $X_{2}=$ $\left\{v_{1}, \ldots, v_{k}\right\}$. If $g=3$, then $R$ is partitioned into neighborhoods of size $k$ by both $X_{1}$ and $X_{2}$. Avoiding 4-cycles ensures that no two vertices of $R$ have the same neighbors in $X_{1}$ and $X_{2}$. Let $w_{r, s}$ be the common neighbor of $u_{r}$ and $v_{s}$ in $R$.

If $g=5$, then matchings join $X_{4}$ to both $X_{1}$ and $X_{2}$, by Lemma 5.5. Index $X_{1}$ and $X_{2}$ so that $u_{j}$ and $v_{j}$ have a common neighbor called $w_{j, j}$ in $X_{4}$. Now $R$ is partitioned into neighborhoods of size $k-1$ by both $X_{1}$ and $X_{2}$. Again let $w_{r, s}$ be the common neighbor of $u_{r}$ and $v_{s}$ in $R$, but now only when $r \neq s$. The vertices of the form $w_{j, j}$ augment $R$ to a set $R^{\prime}$ of size $k^{2}$. When $g=3$, let $R^{\prime}=R$.

Now consider $k=2$. If $g=5$, then the various matchings we have obtained yield an 8-cycle through $u_{1}, w_{1,1}, v_{1}, w_{2,1}, u_{2}, w_{2,2}, v_{2}, w_{1,2}$ in order. We still must add edges joining $R$ to $X_{4}$, but each possible edge creates a triangle. Hence this case does not occur.

If $k=2$ and $g=3$, then $|R|=4$ and $|V(G)|=13$. In Figure 6, let $u_{1}, u_{2}, v_{1}, v_{2}$ be the vertices labeled $12,34,14,23$, respectively; now $w_{1,1}, w_{1,2}, w_{2,2}, w_{2,1}$ are labeled $1,2,3,4$, respectively. Any two vertices of $R$ have a common neighbor in $X_{1} \cup X_{2} \cup X_{3}$, so $P_{3} \nsubseteq \subseteq G[R]$. With no 4-cycles, distance 2 between vertices of $X_{i}$ and $R$ requires exactly one of (a) an edge in $X_{i}$, or (b) a matching in $R$ joining the neighbors of one vertex of $X_{i}$ to the neighbors of 
the other. If each $X_{i}$ induces an edge, then now $G=F_{3}$. Otherwise, $R$ induces a matching, which forbids edges in two of $X_{1}, X_{2}, X_{3}$ and requires an edge in the third; by symmetry, assume $u_{1} u_{2} \in E(G)$. Now $G \cong F_{3}$ by an automorphism sending $\left\{u_{1}, u_{2}, x_{1}\right\}$ to $\left\{x_{3}, x_{2}, x_{1}\right\}$.

The remaining case is $k \geq 3$; we show $a_{t}(G)=1$. Recall that $w_{i, j}$ is adjacent to $u_{i}$ and $v_{j}$. First $x_{1}$ acquires the weight from $X_{1}$ and almost all $w_{i, j}$ with $j \leq i$ via a weakening of the $x_{1}$-greedy protocol. That is, $x_{1}$ acquires weight from the following: (1) $u_{1},(2) u_{2}$ and $w_{2,1}$, and (3) for $3 \leq i \leq k$, all of $u_{i}$ and $\left\{w_{i, j}: j \leq i\right\}$. Note that $x_{1}$ acquires enough weight to permit each subsequent step. After this phase, $x_{1}$ has weight $(k+2)(k+1) / 2-2$.

Next $x_{2}$ acquires the weight from $X_{2}$ and almost all the remaining weight on $R^{\prime}$. That is, $x_{2}$ acquires weight from the following: (1) $v_{1}$, (2) $v_{2}$ and $w_{2,2}$, and (3) for $3 \leq j \leq k$, all of $v_{j}$ and $\left\{w_{i, j}: j>i\right\}$. After this phase, $x_{2}$ has weight $k(k+1) / 2+1$.

Besides $x_{1}$ and $x_{2}$, weight remains on $w_{1,1}, w_{1,2}, x_{3}$, and $X_{3}$. If $g=5$, then weight also remains on $x_{4}, x_{5}$, and $X_{5}$ (note that $X_{4} \subset R^{\prime}$ when $g=5$ ). Since $w_{1,1}$ and $w_{1,2}$ have $u_{1}$ as a common neighbor, their neighbors in $X_{3}$ are distinct; move their weight to $X_{3}$. Since $k \geq 3$, there is another vertex in $X_{3}$; move its weight to $x_{3}$. Now $x_{3}$ can aquire the remaining weight from $X_{3}$, reaching weight $k+3$.

Note that $k(k+1) / 2+1>k+3$, so $x_{2}$ can acquire the weight from $x_{3}$. If $g=5$, then next $x_{5}$ acquires the weight from $x_{4}$ and $X_{5}$, reaching weight $k+2$, and $x_{1}$ can acquire this weight since $(k+2)(k+1) / 2-2>k+2$. Whether $g$ is 3 or 5 , end by combining $x_{1}$ and $x_{2}$.

\section{Operations on Graphs}

We now consider the effect of edge deletion on the acquisition number. We show that the effect can be large, as there are graphs $G$ having an edge $e$ such that $a_{t}(G)=1$ and $a_{t}(G-e) \in \Theta(\sqrt{|V(G)|})$. We will also show that always $a_{t}(G-e)-a_{t}(G) \in O(\sqrt{|V(G)|})$.

We begin with a lemma about the amount of weight that can move to one vertex.

Lemma 6.1. Let $T$ be a tree, with $v \in V(T)$, and suppose some acquisition protocol puts weight $w$ on $v$. If $1 \leq k \leq w$, then there is a protocol that puts weight $k$ on $v$.

Proof. We use induction on $k$. The base case $k=1$ is clear. Also, the case $k=w$ is given, so assume $k<w$.

Let $\mathcal{A}$ be a protocol that yields weight $w$ on $v$. Let $v_{1}, \ldots, v_{t}$ be the neighbors of $v$ that transmit weight to $v$, indexed by their order in $\mathcal{A}$, and let $w_{i}$ be the amount of weight sent from $v_{i}$ to $v$. Let $j$ be the largest integer such that $1+\sum_{i=1}^{j} w_{i} \leq k$, and let $k^{\prime}=$ $k-1-\sum_{i=1}^{j} w_{i}$. Let $T^{\prime}$ be the component of $T-v v_{j+1}$ containing $v_{j+1}$.

The restriction of $\mathcal{A}$ to $T^{\prime}$ puts weight $w_{j+1}$ on $v_{j+1}$, since weight does not move to $v_{j+1}$ from $v$. Since $k^{\prime}<k$, the induction hypothesis yields a protocol $\mathcal{A}^{\prime}$ on $T^{\prime}$ putting weight $k^{\prime}$ 
on $v_{j+1}$. To put weight $k$ on $v$, run the restriction of $\mathcal{A}$ on $T-V\left(T^{\prime}\right)$ until it moves weight from $v_{j}$ to $v$, then run $\mathcal{A}^{\prime}$ on $T^{\prime}$, and finally move the resulting weight on $v_{j+1}$ to $v$.

Recall that the rooted acquisition trees are the trees with acquisition number 1.

Lemma 6.2. Let $(T, r)$ be an n-vertex rooted acquisition tree containing an edge rv. If $T^{\prime}$ is the component of $T-r v$ containing $r$, then $a\left(T^{\prime}\right) \leq 2 \sqrt{n}$.

Proof. Index the components of $T-r$ as $T_{1}, \ldots, T_{k}$ so that $\left|V\left(T_{1}\right)\right| \leq \cdots \leq\left|V\left(T_{k}\right)\right|$. Let $v_{i}$ be the root of $T_{i}$, with $v_{q}=v$. By the recursive characterization of rooted acquisition trees, $T_{i}$ is a rooted acquisition tree, so $a\left(T_{i}\right)=1$ for all $i$.

Let $t$ be the least index such that $\left|V\left(T_{t}\right)\right|>\sqrt{n}$, if some such index exists, and otherwise let $t=k+1$. Note that $t \geq k-\sqrt{n}$. Define a total acquisition protocol for $T^{\prime}$ as follows. Transfer all weight from $\bigcup_{i=1}^{q-1} T_{i}$ onto $r$. Next, transfer weight $\left|V\left(T_{i-1}\right)\right|$ from $T_{i}$ to $r$ for $q+1 \leq i \leq t-1$; this is possible by Lemma 6.1. Finally, transfer all weight from $T_{i}$ to $v_{i}$ for $i \geq t$, leaving this weight on $v_{i}$. This protocol establishes the following bound on $a_{t}(T-e)$ :

$$
a_{t}(T-e) \leq 1+\sum_{i=q+1}^{t}\left(\left|V\left(T_{i}\right)\right|-\left|V\left(T_{i-1}\right)\right|\right)+\max \{k-t, 0\} \leq 1+\left|V\left(T_{t-1}\right)\right|+\sqrt{n} \leq 2 \sqrt{n}
$$

We now consider deleting an arbitrary edge in a rooted acquisition tree.

Lemma 6.3. If $(T, r)$ is an $n$-vertex rooted acquisition tree and $e \in E(T)$, then $a(T-e) \leq$ $1+c \sqrt{n}$, where $c=\frac{2 \sqrt{2}}{\sqrt{2}-1}<6.84$.

Proof. Let $x_{0}$ be the endpoint of $e$ whose distance to $r$ is greater. Let the vertices of the $x_{0}, r$-path in $T$ be $x_{0}, \ldots, x_{k}$, with $r=x_{k}$. Let $T^{\prime}=T-\left\{x_{j-1} x_{j}: 1 \leq j \leq k\right\}$ and let $T_{i}$ be the component of $T^{\prime}$ containing $x_{i}$ (see Figure 7). Since $T^{\prime}$ is a spanning subgraph of $T-e$, we have $a_{t}(T-e) \leq a_{t}\left(T^{\prime}\right)$.

For $0 \leq i \leq k-1$, let $S_{i}$ be the component of $T-x_{i} x_{i+1}$ containing $x_{i}$, and let $S_{k}=T$. By the recursive definition, $\left(S_{i}, x_{i}\right)$ is a rooted acquisition tree, for each $i$. Thus $a_{t}\left(T_{0}\right)=1$. For $1 \leq i \leq k$, Lemma 6.2 , allows all the weight from $T_{i}$ to be moved to at most $2 \sqrt{\left|V\left(S_{i}\right)\right|}$ vertices ( $T_{i}$ plays the role of $T^{\prime}$ in the statement of Lemma 6.2). Therefore

$$
a_{t}\left(T^{\prime}\right) \leq 1+\sum_{i=1}^{k} 2 \sqrt{\left|V\left(S_{i}\right)\right|}
$$

Since $\left(S_{i}, x_{i}\right)$ is a rooted acquisition tree, $\left|V\left(S_{i-1}\right)\right| \leq \frac{1}{2}\left|V\left(S_{i}\right)\right|$. With $\left|V\left(S_{k}\right)\right|=n$, it follows that $\left|V\left(S_{i}\right)\right| \leq n / 2^{k-i}$. Thus we establish the following bound on $a_{t}(T)$ : 


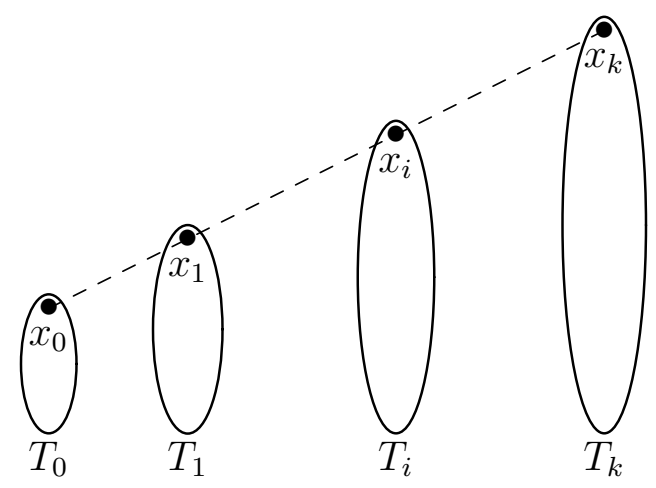

Figure 7: The subgraph $T^{\prime}$ of $T$.

$$
a_{t}(T) \leq a_{t}(T-e) \leq 1+\sum_{i=1}^{k} 2 \sqrt{\frac{n}{2^{k-i}}} \leq 1+2 \sqrt{n}\left(\frac{\sqrt{2}}{\sqrt{2}-1}\right)
$$

The bound extends to all graphs.

Corollary 6.4. If $e$ is an edge of an n-vertex graph $G$, then $a_{t}(G-e) \leq a_{t}(G)+6.84 \sqrt{n}$.

Proof. If $a_{t}(G)=k$, then $G$ contains $k$ acquisition trees $T_{1}, \ldots, T_{k}$ that together span $V(G)$. If $e$ does not belong to any of these trees, then $a_{t}(G-e)=a_{t}(G)$. If $e \in E\left(T_{i}\right)$, then $a_{t}\left(T_{i}-e\right) \leq 6.84 \sqrt{n}+1$ from Lemma 6.3 , and $a_{t}\left(T_{j}\right)=1$ for $j \neq i$.

The order of growth in these upper bounds cannot be reduced.

Theorem 6.5. For each positive integer $n$, there is an $n$-vertex rooted acquisition tree $T$ having an edge $e$ such that $a_{t}(T-e) \geq \sqrt{n} / 2$.

Proof. Let $\ell=\lceil\lg \sqrt{n}\rceil$ and $m=\left\lceil n / 2^{\ell}\right\rceil-1$. We construct a tree $T$ of diameter 4 with central vertex $r$. The neighbors of $r$ are $v_{1}, \ldots, v_{\ell+m}$. For $1 \leq i \leq \ell$, vertex $v_{i}$ has degree $2^{i-1}$. The total number of vertices in the subtree consisting of $r$, its children $v_{1}, \ldots, v_{\ell}$, and their leaf neighbors is $2^{\ell}$. For $i>\ell$, vertex $v_{i}$ has degree $2^{\ell}$ or $2^{\ell}-1$, chosen so $T$ has $n$ vertices. By construction, the $r$-greedy protocol on $T$ transfers all weight to $r$, so $a_{t}(T)=1$.

Let $e=r v_{1}$; we show $a_{t}(T-e) \geq \sqrt{n} / 2$. Let $\mathcal{A}$ be an optimal protocol on $T-e$. If no weight moves from $v_{i}$ to $r$ for $i>\ell$, then $a_{t}(T-e) \geq m+1$, since $v_{1}$ is isolated in $T-e$. Since $m=\left\lceil n / 2^{\ell}\right\rceil-1$ and $\ell=\lceil\lg \sqrt{n}\rceil$, we have $a_{t}(T-e) \geq \sqrt{n} / 2$ in this case.

If $\mathcal{A}$ transfers weight from $v_{i}$ to $r$ for some $i>\ell$, then let $v_{q}$ be the first such vertex. Since $v_{1}$ is not available, $r$ has only $\ell-1$ neighbors that can send it weight before all neighbors 
with index greater than $\ell$. Thus the weight on $r$ is at most $2^{\ell-1}$ before $r$ receives weight from $v_{q}$. Hence $v_{q}$ sends weight at most $2^{\ell-1}$ to $r$. Since $d\left(v_{q}\right) \geq 2^{\ell}-1$, at least $2^{\ell-1}-1$ leaf neighbors of $v_{q}$ retain their weight in $\mathcal{A}$. Also $v_{1}$ is isolated in $T-e$, so weight remains on at least $2^{\ell-1}$ vertices. Hence $a_{t}(T-e) \geq \sqrt{n} / 2$.

\section{Graph Products}

In this section we consider the behavior of acquisition number under graph products. Let $G \square H$ and $G \otimes H$ denote the Cartesian product and strong product of $G$ and $H$, respectively. Each has vertex set $V(G) \times V(H)$. In the Cartesian product, $(u, v)$ and $\left(u^{\prime}, v^{\prime}\right)$ are adjacent if (1) $u=u^{\prime}$ and $v v^{\prime} \in E(H)$ or $(2) v=v^{\prime}$ and $u u \in E(G)$. In the strong product, $(u, v)$ and $\left(u^{\prime}, v^{\prime}\right)$ are adjacent if $u^{\prime} \in N_{G}[u]$ and $v^{\prime} \in N_{H}[v]$. For each product, the notation is a picture of the product of $K_{2}$ with itself.

Proposition 7.1. If $G$ and $H$ are graphs, then $a_{t}(G \otimes H) \leq a_{t}(G \square H) \leq a_{t}(G) a_{t}(H)$.

Proof. First, $a_{t}(G \otimes H) \leq a_{t}(G \square H)$ is implied by $G \square H \subseteq G \otimes H$.

To show $a_{t}(G \square H) \leq a_{t}(G) a_{t}(H)$, first run the same optimal protocol in each copy of $G$. Now all the weight in $G \square H$ lies in $a_{t}(G)$ copies of $H$, and the weight on each vertex of a copy of $H$ is the same. In the $a_{t}(G)$ copies of $H$ with positive weight, run an optimal acquisition protocol for $H$. This leaves positive weight on exactly $a_{t}(G) a_{t}(H)$ vertices.

The bounds in Proposition 7.1 can be arbitrarily loose, even for connected graphs.

Proposition 7.2. Let $G_{m}$ be the graph with $3 m$ vertices obtained from a path with vertices $v_{1}, \ldots, v_{2 m}$ in order by giving each odd indexed vertex a leaf neighbor. For $k \in \mathbb{N}$,

$$
a_{t}\left(G_{4 k} \square K_{2}\right) \leq 3 k=\frac{3}{4} a_{t}\left(G_{4 k}\right) a_{t}\left(K_{2}\right)
$$

and

$$
a_{t}\left(G_{2 k} \otimes K_{2}\right) \leq k=\frac{1}{2} a_{t}\left(G_{2 k}\right) a_{t}\left(K_{2}\right) .
$$

Proof. First note that $G_{m}$ has $m$ "added" leaves, any two of which are separated by a vertex of degree 2 adjacent to no leaf. By Lemma 2.1, $a_{t}\left(G_{m}\right) \geq m$. Since the neighbors of these leaves form a dominating set, by Observation 1.1 equality holds. Since $a_{t}\left(K_{2}\right)=1$, we have $a_{t}\left(G_{m}\right) a_{t}\left(K_{2}\right)=m$.

To see that $a_{t}\left(G_{4 k} \square K_{2}\right) \leq 3 k$, delete $k-1$ edges from each copy of $G_{4 k}$ to get $k$ copies of $G_{4} \square K_{2}$. Figure 8 shows one copy of $G_{4} \square K_{2}$ cut into three groups of vertices, each inducing a graph with acquisition number 1 (the copies of $K_{2}$ are not shown). Therefore $a_{t}\left(G_{4 k} \square K_{2}\right) \leq k a_{t}\left(G_{4} \square K_{2}\right) \leq 3 k$. 


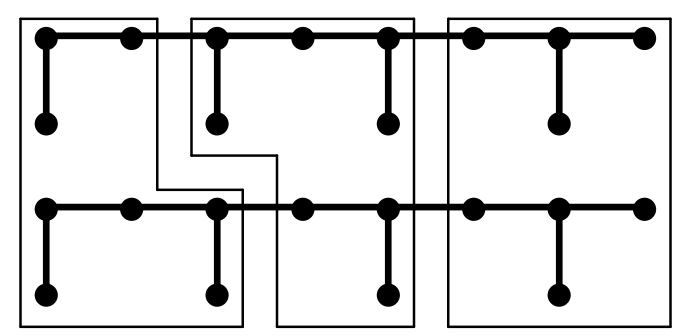

Figure 8: $a_{t}\left(G_{4} \square K_{2}\right) \leq 3$

Finally, note that $a_{t}\left(G_{2 k} \otimes K_{2}\right)$ contains $k$ disjoint copies of $G_{2} \otimes K_{2}$, each of which has acquisition number 1 , so $a_{t}\left(G_{2 k} \otimes K_{2}\right) \leq k$.

We know of only finitely many $G$ and $H$ such that $a_{t}(G \otimes H)<\frac{1}{2} a_{t}(G) a_{t}(H)$. For example, $a_{t}\left(C_{5} \otimes C_{5}\right)=1$, while $a_{t}\left(C_{5}\right) a_{t}\left(C_{5}\right)=4$. For both the Cartesian product and the strong product, it remains open how small $a_{t}$ can be as a function of $a_{t}(G)$ and $a_{t}(H)$.

\section{References}

[1] G. Bacsó, and Z. Tuza, Graphs without induced $P_{5}$ and $C_{5}$. Discuss. Math. Graph Theory 24 (2004), 503-507.

[2] J. A. Bondy, P. Erdős, and S. Fajtlowicz, Graphs of diameter two with no 4-circuits. Discrete Math. 200 (1999), 21-25.

[3] L. Cai, The recognition of union trees. Inform. Process. Lett. 45 (1993), 279-283.

[4] A. J. Hoffman and R. R. Singleton, On Moore graphs with diameters 2 and 3. IBM J. Res. Develop. 4 (1960), 497-504.

[5] D. E. Lampert and P. J. Slater, The acquisition number of a graph. Congr. Numer. 109 (1995), 203-210.

[6] T. D. LeSaulnier and D. B. West, Acquisition-extremal graphs, Discrete Applied Mathematics, Available online 29 January 2013, ISSN 0166-218X, 10.1016/j.dam.2012.12.014.

[7] K. G. Milans, C. J. Stocker, D. B. West, and L. Wiglesworth, Game acquisition number, in preparation.

[8] O. Ore, Note on Hamilton circuits. Amer. Math. Monthly, 67 (1960), 55.

[9] N. Prince, P. S. Wenger, and D. B. West, Unit acquisition number, preprint. 
[10] P. J. Slater, and Y. Wang, The competitive-acquisition numbers of paths. Congr. Numer. 167 (2004), 33-43.

[11] P. J. Slater, and Y. Wang, Some results on acquisition numbers. J. Combin. Math. Combin. Comput. 64 (2008), 65-78.

[12] P. S. Wenger, Fractional acquisition in graphs, preprint. 